\title{
The Cardiac Society of Australia and New Zealand Position Statement on the Diagnosis and Management of Arrhythmogenic Right Ventricular Cardiomyopathy (2019 Update)
}

\section{Christian Hamilton-Craig, MBBS, PhD, FCSANZ ${ }^{\text {h, }{ }^{*} \text {, }}$ Andrew McGavigan, MBChB, MD, FCSANZ ${ }^{a}$, Chris Semsarian, MBBS, PhD, FRACP $^{\mathrm{b}}$, Andrew Martin, MBChB, FRACP ${ }^{\mathrm{c}}$, John Atherton, MBBS, PhD, FRACP ${ }^{d}$, Tony Stanton, MBChB, PhD, FCSANZ ${ }^{e}$, Andre La Gerche, MBBS, PhD, FCSANZ ${ }^{\mathrm{f}}$, Andrew J. Taylor, MBBS, PhD, FCSANZ ${ }^{\mathrm{g}}$, Haris Haqqani, MBBS(Hons), PhD, FCSANZ ${ }^{\mathrm{h}}$}

\footnotetext{
${ }^{\text {a}}$ Flinders Medical Centre and Flinders University, Adelaide, SA, Australia

${ }^{\mathrm{b}}$ Agnes Ginges Centre for Molecular Cardiology at Centenary Institute, The University of Sydney, Sydney, NSW, Australia

${ }^{\mathrm{c}}$ Auckland District Hospital Board, New Zealand

${ }^{\mathrm{d}}$ The Royal Brisbane Hospital, University of Queensland, Brisbane, Qld, Australia

'Sunshine Coast University Hospital, Griffith University, Brisbane, Qld, Australia

${ }^{\mathrm{f}}$ The Baker IDI Institute, University of Melbourne, Melbourne, Vic, Australia

gThe Alfred Hospital, University of Melbourne, Melbourne, Vic, Australia

${ }^{\mathrm{h}}$ The Prince Charles Hospital, University of Queensland, Brisbane, Qld, Australia

${ }^{i}$ Griffith University School of Medicine and The University of Queensland, Brisbane, Australia
}

Keywords Arrythmogenic right ventricular cardiomyopathy $\bullet$ Arrhythmia $\bullet$ Sudden cardiac death $\bullet$ Genetics

\section{Key Points}

1 Arrhythmogenic right ventricular cardiomyopathy (ARVC) is a disease affecting the right, and/or the left ventricle, and commonly presents as an electrical disorder with ventricular arrhythmias and increased risk of sudden cardiac death.

2 ARVC cannot be diagnosed by a single test, and is a clinical diagnosis supported by electrophysiologic, morpho-functional, genetic, and histological data based on the 2010 Task Force 2 criteria.

3 Familial involvement occurs in more than $50 \%$, and referral to a specialist centre with access to genotyping is appropriate in selected cases.
4 Implantable cardioverter-defibrillator (ICD) therapy carries risk, and appropriate thresholds should apply before offering this, particularly in younger individuals.

\section{Clinical Characteristics}

\section{Definition and Prevalence}

Arrhythmogenic right ventricular cardiomyopathy (ARVC) is an inherited myocardial disorder characterised by apoptotic loss of cardiomyocytes and their replacement with fibrofatty tissue [1]. Although the right ventricle (RV) is preferentially and predominantly affected, the left ventricle (LV) is

Abbreviations: ARVC, arrythmogenic right ventricular cardiomyopathy; RV, right ventricle; LV, left ventricle; VA, ventricular arrhythmias; VT, ventricular tachycardia; TAPSE, tricuspid annular plane systolic excursion; EACVI, European Association of Cardiovascular Imaging; TFC, Task Force Criteria; CMR, cardiovascular magnetic resonance; CT, computed tomography; ICD, implantable cardioverter-defibrillator

*Corresponding author. Email: christian.hamilton-craig@health.qld.gov.au

Crown Copyright @ 2019 Published by Elsevier B.V. on behalf of Australian and New Zealand Society of Cardiac and Thoracic Surgeons (ANZSCTS) and the Cardiac Society of Australia and New Zealand (CSANZ). All rights reserved. 
also commonly involved [2]. Recently, the European Association of Cardiovascular Imaging (EACVI) has recommended renaming this entity as "Arrhythmogenic Cardiomyopathy" (AC) in recognition of the biventricular disease expression [3]. However, the term, AC, has not yet become widely adopted, and, for the sake of consistency with previous guidelines, the term ARVC will be used herein. The prevalence of ARVC is highly variable in different geographies, with estimates of between 1 in 2,000 and 1 in 5,000 [3,4]. Our understanding of the disease continues to evolve in parallel with wider recognition of the condition, more comprehensive phenotypic characterisation and deeper understanding of the underlying genetic mechanisms.

\section{Clinical Presentation}

As implied by its name, ARVC is first and foremost a disease associated with the development of significant ventricular arrhythmias (VA). It most commonly presents with palpitations and syncope, but can also become first manifest as sudden death or resuscitated cardiac arrest. The onset of symptoms is usually between 20 and 40 years of age, but cases can occur in childhood. Unlike other genetic cardiomyopathies such as hypertrophic cardiomyopathy (HCM), late presentation does not confer a benign prognosis [5]. The male: female ratio is about 3:1 at presentation, but in genotyped cohorts the sex ratio is unity. While the clinical course is usually progressive, the rate is highly variable. Conventionally four stages have been described, (latent, symptomatic ventricular arrhythmias, isolated right heart failure, dilated biventricular cardiomyopathy) but recent studies employing late enhancement magnetic resonance support a biventricular pathology in the majority of patients from the outset [6]. Certain regions of the right ventricle are classically affected, the so-called triangle of dysplasia (outflow tract, apex, sub-tricuspid area). The disease process begins in the epicardium and progresses inwards in most cases. ARVC has been unusually prominent as a cause of death in young athletes in Italy, with a particular concentration in the Veneto region. There is strong evidence that ARVC is a desmosomal disease, and this would accord with the thinner walled RV initially showing morphologic change, especially in athletes or other individuals with high exercise levels in whom there is some evidence that symptoms and sudden death present at a younger age [7]. Knowledge of the responsible genetic mutations is presently incomplete and their relation to the phenotype is likely complex. The writing group was comprised of representative members of the CSANZ Councils, and approved by the CSANZ Quality \& Safety Committee (see Governance section).

\section{Clinical Diagnosis}

Arrhythmogenic right ventricular cardiomyopathy cannot be diagnosed by a single test, and is a clinical diagnosis supported by electrophysiologic, morpho-functional, genetic, and histological criteria. The initial Task Force criteria (TFC) (see below) were agreed in 1994 but lacked sensitivity for affected family members and possibly asymptomatic younger people at increased risk of sudden death. The 2010 modified Task Force criteria have improved sensitivity (Table 1) [4,8]. These modified guidelines are more prescriptive for defining morphologic RV changes with separate criteria for echocardiographic and magnetic resonance imaging (MRI) assessments. The diagnostic terminology for the revised task force criteria are:

Definite diagnosis: Two (2) major or one major and two minor criteria or four minor from different categories;

Borderline: One (1) major and one minor or three minor criteria from different categories;

Possible: One (1) major or two minor criteria from different categories.

\section{Electrophysiologic Criteria}

It is important to emphasise that ARVC is a disease phenotypically characterised by significant arrhythmias and disturbances of normal ventricular electrophysiology. Imaging abnormalities in isolation, particularly in the absence of a reasonable pre-test probability, are unlikely to represent sufficient grounds to correctly diagnose this condition according to Revised Task Force Criteria [8]. Patients presenting with VA need detailed expert assessment to work through the mechanism and differential diagnosis of the underlying condition, particularly to exclude a scar-related re-entrant aetiology. In the absence of structural heart disease, idiopathic VT, often due to a focus of triggered activity in the outflow tracts is a possible diagnostic consideration [9]. ARVC can present with similar ventricular tachycardia (VT) at an early stage when there is limited and patchy structural disease only, making diagnosis difficult. Conversely, in highlevel athletes with VT, it can be extremely difficult to exclude ARVD in the presence of the ventricular remodelling often seen in this group [7].

Given its predilection to cause significant ventricular fibrosis, symptoms related to scar-related VAs, such as rapid palpitations and syncope, are the most frequent mode of presentation of patients with ARVC. Frequent ventricular ectopy and non-sustained VAs are also common. The majority of ventricular tachycardia (VT) in this condition has left bundle branch block (LBBB) morphology reflecting its origin from the right ventricle. Patients with focal idiopathic outflow tract VT in a setting of structurally normal hearts have inferior frontal plane QRS axis. However, this morphology can also be seen in ARVD, particularly exercise-induced VTs due to triggered activity, and hence forms a Minor Criterion. Ventricular tachycardia exhibiting LBBB configuration with superior axis comprises a Major Criteria given that this is not a typical pattern for idiopathic VTs. Frequent ventricular ectopy forms a Minor Criteria but it should be noted that complex ectopy induced by high-dose isoprenaline challenge represents an emerging diagnostic investigation in ARVC [10].

There are several characteristic abnormalities seen on electrocardiograph (ECG) during sinus rhythm that are suggestive of ARVC [Figure 1]. These reflect abnormalities of repolarisation and/or depolarisation. Although no ECG pattern is pathognomonic of the disease, and an abnormal ECG 


\section{Table 1 Updated Task Force Criteria (2010) for ARVD/C.}

\section{Original task force criteria}

I. Global or regional dysfunction and structural alterations

\section{Major}

- Severe dilatation and reduction of RV ejection

fraction with no LV impairment

- Localised RV aneurysms

- Severe segmental dilatation of the RV

\section{Minor}

- Mild global RV dilatation and/or ejection

fraction reduction with normal LV

- Mild segmental dilatation of the RV

- Regional RV hypokinesis

II. Tissue characterisation of wall

Major

- Fibrofatty replacement of myocardium on endomyocardial biopsy

Minor -

III. Repolarisation abnormalities

Major -

\section{Minor}

- Inverted $\mathrm{T}$ waves in right precordial leads (V2 and V3) (people age $>12$ years, in absence of right bundle-branch block)
Revised task force criteria

\section{Major}

By 2D echo:

Regional RV akinesia, dyskinesia or aneurysm and 1 of the following (end diastole and corrected for body size (PLAX/BSA)):

- PLAX RVOT $>32 \mathrm{~mm}$ PLAX/BSA $>19 \mathrm{~mm} / \mathrm{m}^{2}$

- PSAX RVOT $>36 \mathrm{~mm}$ PSAX/BSA $>21 \mathrm{~mm} / \mathrm{m}^{2}$

- Fractional area change $<33 \%$

By MRI:

Regional RV akinesia or dyskinesia or dyssynchrounous RV contraction and 1 of the following:

- Ratio of RV EDV to BSA $>110 \mathrm{ml} / \mathrm{m}^{2}$ (male) or $>100 \mathrm{ml} / \mathrm{m}^{2}$ (female)

- RV ejection fraction $<40 \%$

Minor

By 2D echo:

Regional RV akinesia or dyskinesia and 1 of the following (end diastole and corrected for body size (PLAX/BSA))

- PLAX RVOT $>29$ to $<32 \mathrm{~mm}$ PLAX/BSA $>16$ to $<19 \mathrm{~mm} / \mathrm{m}^{2}$

- PSAX RVOT $>32$ to $36 \mathrm{~mm}$ PSAX/BSA $>18$ to $<21 \mathrm{~mm} / \mathrm{m}^{2}$

- Fractional area change $>33 \%$ to $<40 \%$

By MRI:

Regional RV akinesia or dyskinesia or dyssynchrounous RV contraction and 1 of the following:

- Ratio of RV EDV to BSA $>100$ to $<110 \mathrm{ml} / \mathrm{m}^{2}$ (male) or $>90$ to

$<100 \mathrm{ml} / \mathrm{m}^{2}$ (female)

- RV ejection fraction $>40 \%$ to $<45 \%$

\section{Major}

- Residual myocytes $60 \%$ by morphometric analysis (or $50 \%$ if estimated) with fibrous replacement of the RV free wall myocardium in $>1$ sample, with or without fatty replacement of tissue on endomyocardial biopsy

\section{Minor}

- Residual myocytes $60 \%$ to $75 \%$ by morphometric analysis (or $50 \%$ to $65 \%$ if estimated), with fibrous replacement of the RV free wall myocardium in $>1$ sample, with or without fatty replacement of tissue on endomyocardial biopsy

\section{Major}

- Inverted T waves in right precordial leads (V1, V2, and V3) or beyond in individuals 14 years of age (in the absence of complete right bundle-branch block QRS $>120 \mathrm{~ms}$ )

\section{Minor}

- Inverted T waves in leads V1 and V2 in individuals 14 years of age (in the absence of complete right bundle-branch block) or in V4, V5, or V6

- Inverted T waves in leads V1, V2, V3, and V4 in individuals 14 years of age in the presence of complete right bundle-branch block 


\author{
IV. Depolarisation/conduction abnormalities \\ Major \\ - Epsilon waves or localised prolongation $(0.110$ \\ ms) of the QRS complex in right precordial leads \\ (V1 to V3) \\ Minor \\ - Late potentials (SAECG)
}

\section{Arrhythmias \\ Major -}

\section{Minor}

- Left bundle-branch block-type ventricular tachycardia (sustained and nonsustained)

- Frequent ventricular extrasystoles (1,000 per

$24 \mathrm{hrs})$

VI. Family history

Major

- Familial disease confirmed at necropsy or surgery

\footnotetext{
Minor

- Family history of premature sudden death (35 years of age) due to suspected ARVC/D

- Familial history (clinical diagnosis based on present criteria)
}

\author{
Major \\ - Epsilon wave (reproducible low-amplitude signals between end of \\ QRS complex to onset of the $\mathrm{T}$ wave) in the right precordial leads \\ (V1 to V3) \\ Minor \\ - Late potentials by SAECG in $>1$ of 3 parameters in the absence of a \\ QRS duration of $>110 \mathrm{~ms}$ on the standard ECG \\ - Duration of terminal QRS $<40 \mathrm{mV}>38 \mathrm{~ms}$ \\ - Root-mean-square voltage of terminal $40 \mathrm{~ms}<20 \mathrm{mV}$ \\ - Terminal activation duration of QRS $>55 \mathrm{~ms}$ measured from nadir \\ of the $\mathrm{S}$ wave to the end of the QRS including the R1 in V1, V2 or V3 \\ in the absence of complete right bundle branch block
}

\author{
Major \\ - Nonsustained or sustained ventricular tachycardia of left bundle- \\ branch morphology with superior axis (negative or indeterminate \\ QRS in leads II, III, and aVF and positive in lead aVL)

\section{Minor} \\ - Nonsustained or sustained ventricular tachycardia of RV outflow \\ configuration, left bundle-branch block morphology with inferior axis \\ (positive QRS in leads II, III, and aVF and negative in lead aVL) or of \\ unknown axis \\ - >500 ventricular extrasystoles per $24 \mathrm{hrs}$
}

\author{
Major \\ - ARVC/D confirmed in a first-degree relative who meets current \\ Task Force criteria \\ - ARVC/D confirmed pathologically at autopsy or surgery in a first- \\ degree relative \\ - Identification of a pathogenic mutation categorized as associated or \\ probably associated with ARVC/D in the patient \\ Minor \\ - History of ARVC/D in a first-degree relative in whom it is not \\ possible or practical to determine whether the family member meets \\ current Task Force criteria \\ - Premature sudden death (35 years of age) due to suspected ARVC/ \\ $\mathrm{D}$ in a first-degree relative \\ - ARVC/D confirmed pathologically or by current Task Force \\ Criteria in second-degree relative
}

Abbreviations: PLAX, parasternal long-axis view; RVOT, right ventricle outflow tract; BSA, body surface area; PSAX, parasternal short-axis view; aVF, augmented voltage unipolar left foot lead; aVL, augmented voltage unipolar left arm lead; ARVC, arrythmogenic right ventricular cardiomyopathy; RV, right ventricular; LV, left ventricular; 2D, two dimensional; SAECG, signal-averaged electrocardiography; MRI, magnetic resonance imaging.

Hypokinesis is not included in this or subsequent definitions of RV regional wall motion abnormalities for the revised criteria.

A pathogenic mutation is a DNA alteration associated with ARVC/D that alters or is expected to alter the encoded protein, is unobserved or rare in a large nonARVC/D control population, and either alters or is predicted to alter the structure or function of the protein or has demonstrated linkage to the disease phenotype in a conclusive pedigree.

Source: Marcus FI, et al: Diagnosis of arrhythmogenic right ventricular cardiomyopathy/dysplasia proposed modification of the task force criteria [4].

is not necessarily a part of the phenotype, particularly early in the disease course, the greater the degree of abnormality the more likely the diagnosis. This is reflected in the Revised Task Force Criteria, with $\mathrm{T}$ wave inversion in $\mathrm{V} 1-\mathrm{V} 3$ or beyond (in the absence of RBBB) in those $>14$ years of age now being a Major Criterion. When present however, the $\mathrm{T}$ wave inversion in the right precordial leads correlates with RV enlargement and fibro-fatty infiltration and similar inversion in the lateral or inferior leads seems to correlate with LV involvement.

Like these repolarisation abnormalities, significant ventricular fibrosis affects depolarisation in a regional manner [11]. 


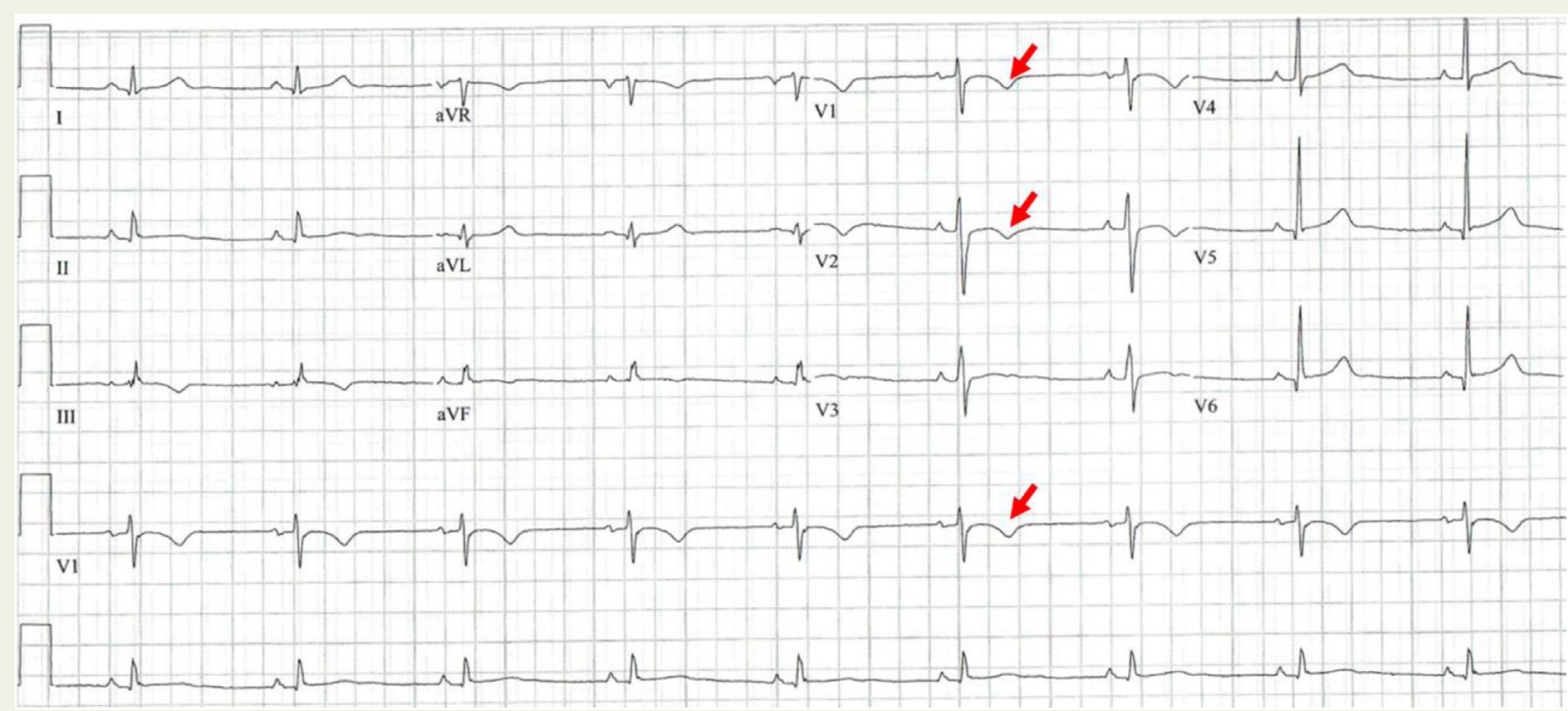

Figure 1 ECG of a patient with genotype-positive ARVC and clinical features of ventricular arrhythmias and confirmatory EP study. Note the T-wave inversion in the anterior leads (particularly V1, V2, red arrows).

Abbreviations: EP, electrophysiology; ECG, electrocardiograph; ARVC, arrythmogenic right ventricular cardiomyopathy.

Delayed and distorted wavefront propagation lead to late potentials occurring in the terminal portion of the QRS. Late potentials may be detected by signal-averaged ECG but their overt presence as an epsilon wave on the surface ECG is usually a late feature [12]. The reproducibility and interobserver agreement of diagnosing the presence of epsilon waves is low [12]. Depolarisation abnormalities related to slowed wavefront conduction in the scarred right ventricle (parietal block) form a Minor criterion, with prolonged S wave duration in the right precordial leads. This is associated with a prolonged total endocardial right ventricular activation time on invasive mapping [13].

\section{Imaging}

Morphologic assessment of the RV is hampered by its architecture and an inadequate knowledge of the limits of normality, so that subtle early changes are easily missed. The preferred imaging modality is MRI and the modified Task Force criteria are more precise in what constitutes minor and major abnormalities. The ability to demonstrate late enhancement with MRI scanning has been an advance and has increased detection of LV involvement, although no provision for this information is presently included in the current Task Force criteria. Biplane right ventricular angiography may also be helpful, however the presence of so-called abnormal findings in the normal population and inter-observer variation limit its diagnostic utility [26]. The only imaging modality at present that can comment on tissue characterisation is MRI. However, there are concerns that fatty infiltration can be overinterpreted and could lead to an overdiagnosis of ARVC [8].

\section{Echocardiography}

Echocardiography is widely accessible and convenient and, for these reasons, it remains an important investigation in both the diagnosis of, and screening for, ARVC. Echo has traditionally struggled to image the RV well due to its anatomical position, challenging acoustic windows, complex geometrical shape and load dependent physiology. The relatively poor sensitivity of echo to detect early disease remains a key clinical consideration. For these reasons, if ARVC is considered clinically likely, echocardiography should not be used as the sole imaging modality.

The hallmarks of ARVC on echo are RV regional wall motion abnormalities, localised RV aneurysms, global RV dysfunction and RV dilatation. The recommendations of the TFC 2010 focussed on standard two dimensional (2D) echo measurements such as the RV outflow tract (RVOT) diameter in both the parasternal long and short axes and calculation of the RV fractional area change (RVFAC) (Table 1) with a combination required to fulfil the diagnostic criteria. Whilst the specificity of these measures is high, their sensitivity is relatively modest given the comparative difficulty in imaging these areas and the fine margins, often millimetres, within the criteria (TFC 2010). Indeed, a 2014 study from the Nordic ARVC Registry demonstrated that just $50 \%$ of ARVC definite individuals who were cardiac magnetic resonance image (CMR) positive for the features of ARVC fulfilled the TFC 2010 echocardiographic criteria [14].

The additional measurements of the RV basal diameter and tricuspid annular plane systolic excursion (TAPSE) from the four-chamber view have been suggested by the EACVI in their recent Expert Consensus Document [3]. This document also considered the improvements in technology since 2010 to incorporate some of the emerging quantitative indices of both the RV and LV.

- Tissue Doppler: Tissue Doppler Derived Tricuspid Lateral Annular Systolic Velocity (TV s') is angle dependent but 
has been shown to correlate well with other measures of global RV function.

- Right ventricular speckle tracking: An RV focussed fourchamber view can be used to measure both RV free wall peak systolic strain (average of the three free wall segments) and RV global peak systolic strain (average of the three free wall segments plus three segments from the interventricular septum). Both have been shown to be reduced in small studies comparing ARVC patients with controls [15]. RV mechanical dispersion, which is more pronounced in ARVC, can also be calculated as the standard deviation of time-to-peak strain [15].

- Right ventricular 3D echo: Published reference values are available for 3D volumes and ejection fraction [16]. RV 3D ejection fraction (EF) of $\leq 40 \%$ is abnormal although there is limited data in the setting of ARVC.

- Left ventricular speckle tracking: LV global longitudinal strain has been shown to be a reliable marker of subclinical LV dysfunction in many conditions, often in the presence of a preserved 2D Simpson's biplane ejection fraction. LV involvement is thought to occur in $50 \%$ of all cases and has shown to be predict adverse outcomes in ARVC [17]. However, changes in left ventricular-global longitudinal strain (LV-GLS) are non-specific and may relate to pathologies other than ARVC.

\section{Cardiac MRI}

Cardiac magnetic resonance (CMR) is the reference standard for evaluation of right ventricular size, systolic function, and presence/absence of wall motion abnormalities. The TFC 2010 include CMR parameters of RV regional dysfunction, reduced RV-EF and enlarged indexed RV end-diastole volume, as well as localised RV wall thinning and presence of micro/macro-aneurysms.

Due to the complex RV geometry, it is important to image all of the RV walls in multiple imaging planes (and not just a single LV short axis series, which is prone to through-plane motion, and which may give rise to the false appearances of micro/macro-aneurysms). Evaluation of the RV is complex, subjective, and requires expert CMR reporters for accurate diagnosis. Significant wall motion abnormalities are akinesis/dyskinesis, but importantly hypokinesis alone is not included in the definition of RV regional wall motion abnormalities in the revised Task Force Criteria [3,4].

The EACVI has issued a recommended CMR Imaging Protocol for evaluation of ARVC (Supplementary Data, Haugaa et al. [3]). Although fibro-fatty infiltration can sometimes be detected by MRI, this is not currently part of the TFC criteria and remains a histopathologic diagnosis, therefore fat imaging by MRI is not routinely recommended.

The presence of late gadolinium enhancement (LGE) on inversion recovery post-contrast imaging may aid in the assessment of patients presenting with ventricular tachycardia, as the cause of VT may relate to other conditions which may be identified on LGE imaging. Patients with Desmoplakin mutations may exhibit early LV involvement with sub-epicardial LGE that can be mistaken for myocarditis [18].
As ARVC is a complex disease entity, with clinical, electrical, and morpho-functional diagnostic aspects, it is recommended that the CMR report describe the RV volumes, presence/absence of regional dyskinesia, and whether the findings form a Minor or Major criterion. Of critical importance, the diagnosis of ARVC cannot be established by CMR alone, thus this diagnosis should not be concluded in the report.

\section{Phenocopies}

Many other conditions (which are far more common than ARVC) may cause RV enlargement, dysfunction, and be associated with arrhythmias (such as atrial or ventricular shunts, anomalous pulmonary venous drainage, RV infarction, primary tricuspid regurgitation). Given the rarity of true ARVC, consideration must be given to these differential diagnoses, imaging should be tailored to exclude these where possible, and appropriate attention paid to the pre-test probability of ARVC in each individual case when interpreting imaging findings.

\section{Computed tomography}

Cardiac computed tomography (CT) is not currently recommended in the routine diagnostic workup of patients with ARVC. However, current generation multidetector computed tomography has high spatial resolution and may allow measurement of RV and LV volumes and systolic function. This may be applicable for patients who have suboptimal echo windows, and contraindications to CMR scanning (such as non-conditional implanted devices). The 2010 ACC/AHA Cardiac CT appropriate use criteria describe Cardiac CT as "appropriate" for the evaluation of structural RV and/or LV remodelling in suspected $\mathrm{AC}$, in particular for those patients in the above context. However, due to the ionising radiation exposure (and, for functional analysis, increased exposure is required using retrospective gating to acquire both systolic and diastolic phases), CT is not recommended as first line for AC.

\section{Histology}

Abnormal histology can contribute a major criterion, but many histopathologists lack sufficient experience to make a confident interpretation. Recently, immunohistochemical analysis of an endomyocardial biopsy sample was reported as a possibly highly sensitive and specific diagnostic test for ARVC. Interestingly, plakoglobin signal levels were reduced in normal-appearing LV as well as areas showing typical fibrofatty change in the RV. Unfortunately, ongoing work suggests this finding may be similarly present in dilated cardiomyopathy so that its specificity and clinical value remain to be determined.

\section{Molecular Genetics}

Familial involvement occurs in more than $50 \%$, but unexpected deaths in family members older than 35 years do not qualify as a Minor criterion. Modified Task Force criteria (one minor criterion only) have been proposed, but not 
universally accepted for family members with a diagnosed proband.

Genetic screening is commercially available in major centres world-wide, and referral to a specialist centre with access to genotyping may be appropriate in selected cases [19]. There are five main desmosomal genes associated with ARVC: plakophilin-2 (PKP2), desmoplakin (DSP), desmoglein (DSG-2), desmocollin (DSC2), and plakoglobin (JUP). Inheritance is autosomal dominant unless otherwise specified. Multiple mutations have been reported to be associated with an increased risk of sudden death. Identification of a variant categorised as pathogenic or likely pathogenic to cause ARVC has now been assigned major criterion status in the revised criteria.

Plakophilin-2 (PKP2) is the most commonly implicated disease gene with mutations reported in $6-43 \%$ of Task Force-positive patients, with penetrance among mutation carriers reported to be nearly $50 \%$. Patients carrying PKP2 mutations present at an earlier age than those without mutations and the arrhythmia-free survival is lower. Desmoglein2 (DSG2) and Desmocollin-2 (DSC2) mutations have been predominantly associated with left ventricular involvement. Desmoplakin (DSP) mutations have been identified in two autosomal recessive syndromes (Carvajal and a "Naxos like" syndrome) and in some patients in the North American ARVC Registry. It is hypothesised that desmoplakin mutations predispose to early left ventricular involvement from disruption of cytoskeletal integrity. Homozygous mutations in Plakoglobin (JUP) are associated with Naxos disease, an autosomal recessive condition characterised by palmo-plantar hyperkeratosis and woolly hair. The transmembrane gene TMEM43 has been implicated in ARVD5 in Newfoundland families and affected males have a median survival of 41 compared to 83 years in mutation-free individuals. Affected males are also three times more likely to develop heart failure. A few families' mutations have been described in transforming growth factor beta 3 (TGF-B3) and cardiac ryanodine receptor $(R Y R 2)$ genes, but their significance seems presently marginal.

The main role of genetic testing in ARVC is both to confirm diagnosis but also in screening family members by cascade genetic testing. Pre- and post- test genetic counselling is recommended in all patients and family members considering genetic testing.

\section{Management}

\section{Affected Individuals}

\section{Exercise}

Strenuous exercise (e.g. basketball, squash, skiing, soccer, singles tennis, cycling, running and windsurfing) is strongly recommended to be avoided. There is a plausible explanation as to why such exercise might be deleterious with the thinner walled right ventricle more vulnerable to cellular disruption and early registry experience would corroborate this view [7].

\section{Implantable Cardioverter-Defibrillator (ICD)}

Recent data have allowed for the production of risk stratification schemes for sudden death in ARVC [20]. The only effective known risk mitigation tool is the ICD. There is consensus that cardiac arrest survivors or patients with sustained VT should have an ICD. There is also acknowledgment of the high risk conferred by ventricular dysfunction, syncope and nonsustained VT, and thus primary prevention ICD should also be recommended to these patients. Patients without identified risk factors or healthy desmosomal gene mutation carriers have no indication for an ICD at present [20].

Particularly in young patients, the lifetime risk of ICD implantation is not trivial. Late ICD system infections, endocarditis, inappropriate shocks, vascular occlusion and lead failure can all occur. The latter may occur in up to $20 \%$ by 10 years [21]. With progressive right ventricular scarring, $R$ wave amplitude sensing may become significantly impaired over time in patients with ARVC [22]. Lead thromboembolism may raise pulmonary pressure and potentially worsen right ventricular function [23]. Lead-related tricuspid valve dysfunction may also hasten right ventricular decline. A signification number of patients may come to require lead extraction over the long-term, a procedure with considerable morbidity and mortality. Given these transvenous leadrelated risks of conventional ICDs, subcutaneous ICDs may have important advantages in this group of patients but come at the cost of a significant inappropriate shock rate, shorter battery longevity and lack of anti-tachycardia pacing that may terminate VT painlessly.

\section{Antiarrhythmic Drugs}

It should be stressed that ICDs are not a treatment for VAs but are implanted to mitigate against the risk of sudden death in ARVC. Implantable cardioverter-defibrillator shocks are painful and thus all patients with ICDs and sustained VT need management of the arrhythmia with either antiarrhythmic drugs or catheter ablation.

Beta blockers, sotalol and amiodarone are the most commonly used antiarrhythmic agents to treat symptomatic ventricular arrhythmias and prevent ICD shocks [24]. There are no data to suggest a prognostic benefit for any of these and extracardiac adverse effects, particularly of amiodarone, can be debilitating and need to be closely monitored for. There is no compelling evidence that beta blocker therapy is disease modifying.

\section{Catheter Ablation}

Due to the limited efficacy and potential adverse effects of antiarrhythmic drugs, catheter ablation is an important therapeutic option for the management of VT in ARVC. The initially disappointing published results were in the context of endocardial ablation only. The disease process in ARVC has a transmural involvement in the majority of patients and involves the epicardium early [1]. When combined epicardial 
and endocardial catheter ablation is performed to map and target all inducible VTs as well as aggressively modify the ventricular substrate, good long-term antiarrhythmic drug free outcomes are possible in the majority of patients [25].

\section{Cardiac Transplantation}

Occasional patients will come to require cardiac transplantation either because of progressive right heart or biventricular failure or rarely, intractable VAs.

\section{Asymptomatic Family Members}

Both a 12-lead and, if possible, a signal-averaged ECG should be obtained on all first-degree relatives. Because of the relative insensitivity of echo, MRI scanning is also recommended for adults and older children beginning age 10-12 years when general anaesthesia is no longer necessary. Screening should be repeated every 5 years until age 30 , and once more at age 40 . After age 40 coronary artery disease is a greater risk for sudden death and history and an ECG will suffice in hitherto normal individuals. If the MRI is minimally abnormal (mild segmental or global enlargement) the scan should be repeated after 3 years, with reversion to 5-yearly thereafter if there is no further interval change. The presence of intramyocardial fat by itself may be normal, but congruent late enhancement on MRI suggests pathology. In younger children, an ECG and an echocardiogram at 3-yearly intervals is recommended until MRI without anaesthesia is feasible. Genetic screening should be undertaken where possible, but caution is warranted in concluding mutation-free individuals are necessarily at low risk until more data are available.

\section{Genetic Counselling}

There is presently insufficient information on what to confidently advise patients on the basis of presently identified mutations. However, genetic testing should be encouraged where the opportunity for analysis exists, to expand our knowledge base and the opportunity for genotype-phenotype correlation. Notwithstanding the potential for sudden death in a minority, it is important to emphasise that most diagnosed patients can lead full and normal lives.

\section{Conclusion}

Arrythmogenic right ventricular cardiomyopathy most commonly presents as an electrical disorder, with complex genetic and morpho-functional aspects. The current Revised Task Force Criteria have improved the diagnosis, with more detailed electrical, as well as functional criteria. Magnetic resonance imaging is an important tool, but the diagnosis cannot be made on MRI metrics alone, and pre-test probability based on electrical and genetic criteria should be considered when interpreting results. Implantable cardioverterdefibrillator therapy carries risk, and appropriate thresholds should apply before offering this, particularly in younger individuals. The Task Force Criteria are likely to be updated in the near future, at which time this document will be reviewed.

\section{Governance and Conflicts of Interest}

The writing group was comprised of representative members of the CSANZ Councils, and the chairs appointed by consensus. Writing began in 2017 and was completed in July 2018 when the document was approved by the CSANZ Quality and Safety Committee (25 January 2019) and referred on to the CSANZ Board. The Position statement will be reviewed in 5 years or earlier if the International Task Force are updated.

\section{Conflicts of Interest}

The authors report no relevant conflicts of interest.

\section{References}

[1] Basso C, Thiene G, Corrado D, Angelini A, Nava A, Valente M. Arrhythmogenic right ventricular cardiomyopathy. Dysplasia, dystrophy, or myocarditis? Circulation 1996;94:983-91.

[2] Marcus F, Towbin JA. The mystery of arrhythmogenic right ventricular dysplasia/cardiomyopathy: from observation to mechanistic explanation. Circulation 2006;114:1794-5.

[3] Haugaa KH, Basso C, Badano LP, Bucciarelli-Ducci C, Cardim N, Gaemperli $\mathrm{O}$, et al. Comprehensive multi-modality imaging approach in arrhythmogenic cardiomyopathy-an expert consensus document of the European Association of Cardiovascular Imaging. Eur Heart J Cardiovasc Imaging 2017;18:237-53.

[4] Marcus FI, McKenna WJ, Sherrill D, Basso C, Bauce B, Bluemke DA, et al. Diagnosis of arrhythmogenic right ventricular cardiomyopathy/dysplasia: proposed modification of the Task Force Criteria. Eur Heart J 2010;31:806-14.

[5] Bhonsale A, Te Riele A, Sawant AC, Groeneweg JA, James CA, Murray B, et al. Cardiac phenotype and long-term prognosis of arrhythmogenic right ventricular cardiomyopathy/dysplasia patients with late presentation. Heart Rhythm 2017;14:883-91.

[6] te Riele AS, Bhonsale A, James CA, Rastegar N, Murray B, Burt JR, et al Incremental value of cardiac magnetic resonance imaging in arrhythmic risk stratification of arrhythmogenic right ventricular dysplasia/cardiomyopathyassociated desmosomal mutation carriers. J Am Coll Cardiol 2013;62:1761-9.

[7] James CA, Bhonsale A, Tichnell C, Murray B, Russell SD, Tandri H, et al Exercise increases age-related penetrance and arrhythmic risk in arrhythmogenic right ventricular dysplasia/cardiomyopathy-associated desmosomal mutation carriers. J Am Coll Cardiol 2013;62:1290-7.

[8] Femia G, Sy RW, Puranik R. Systematic review: Impact of the new task force criteria in the diagnosis of arrhythmogenic right ventricular cardiomyopathy. Int J Cardiol 2017;241:311-7.

[9] Santangeli P, Pieroni M, Dello Russo A, Casella M, Pelargonio G, Macchione A, et al. Noninvasive diagnosis of electroanatomic abnormalities in arrhythmogenic right ventricular cardiomyopathy. Circ Arrhythm Electrophysiol 2010;3:632-8

[10] Denis A, Sacher F, Derval N, Martin R, Lim HS, Pambrun T, et al. Arrhythmogenic response to isoproterenol testing vs. exercise testing in arrhythmogenic right ventricular cardiomyopathy patients. Europace 2018;20:f30-6. http://dx.doi.org/10.1093/europace/euy007.

[11] Tschabrunn CM, Haqqani HM, Santangeli P, Zado ES, Marchlinski FE. 12-lead electrocardiogram to localize region of abnormal electroanatomic substrate in arrhythmogenic right ventricular cardiomyopathy. JACC Clin Electrophysiol 2017;3:654-65.

[12] Platonov PG, Calkins H, Hauer RN, Corrado D, Svendsen JH, Wichter T, et al. High interobserver variability in the assessment of epsilon waves: implications for diagnosis of arrhythmogenic right ventricular cardiomyopathy/dysplasia. Heart Rhythm 2016;13:208-16. 
[13] Tandri H, Asimaki A, Abraham T, Dalal D, Tops L, Jain R, et al. Prolonged RV endocardial activation duration: a novel marker of arrhythmogenic right ventricular dysplasia/cardiomyopathy. Heart Rhythm 2009;6:769-75.

[14] Borgquist R, Haugaa KH, Gilljam T, Bundgaard H, Hansen J, Eschen O, etal. The diagnostic performance of imaging methods in ARVC using the 2010 Task Force criteria. Eur Heart J Cardiovasc Imaging 2014;15:1219-25.

[15] Sarvari SI, Haugaa KH, Anfinsen OG, Leren TP, Smiseth OA, Kongsgaard E, et al. Right ventricular mechanical dispersion is related to malignant arrhythmias: a study of patients with arrhythmogenic right ventricular cardiomyopathy and subclinical right ventricular dysfunction. Eur Heart J 2011;32:1089-96.

[16] Maffessanti F, Muraru D, Esposito R, Gripari P, Ermacora D, Santoro C, et al. Age-, body size-, and sex-specific reference values for right ventricular volumes and ejection fraction by three-dimensional echocardiography: a multicenter echocardiographic study in 507 healthy volunteers. Circ Cardiovasc Imaging 2013;6:700-10.

[17] Mast TP, Teske AJ, vd Heijden JF, Groeneweg JA, Te Riele AS, Velthuis $\mathrm{BK}$, et al. Left ventricular involvement in arrhythmogenic right ventricular dysplasia / cardiomyopathy assessed by echocardiography predicts adverse clinical outcome. J Am Soc Echocardiogr 2015;28:1103-1113 e9.

[18] Abidov A, Kannan A, Oliva IB, Marcus FI. CMR features of ARVC/D. In: Marcus FI, editor. Cardiac MRI in diagnosis, clinical management, and prognosis of arrhythmogenic right ventricular cardiomyopathy/dysplasia. Academic Press; 2016.

[19] Hershberger RE, Givertz MM, Ho CY, Judge DP, Kantor PF, McBride KL, et al. Genetic evaluation of cardiomyopathy - a heart failure society of america practice guideline. J Card Fail 2018;24:281-302.
[20] Calkins H, Corrado D, Marcus F. Risk stratification in arrhythmogenic right ventricular cardiomyopathy. Circulation 2017;136:2068-82.

[21] Kleemann T, Becker T, Doenges K, Vater M, Senges J, Schneider S, et al. Annual rate of transvenous defibrillation lead defects in implantable cardioverter-defibrillators over a period of $>10$ years. Circulation 2007;115:2474-80.

[22] Herman AR, Gardner M, Steinberg C, Yeung-Lai-Wah JA, Healey JS, Leong-Sit $\mathrm{P}$, et al. Long-term right ventricular implantable cardioverterdefibrillator lead performance in arrhythmogenic right ventricular cardiomyopathy. Heart Rhythm 2016;13:1964-70.

[23] Supple GE, Ren JF, Zado ES, Marchlinski FE. Mobile thrombus on device leads in patients undergoing ablation: identification, incidence, location, and association with increased pulmonary artery systolic pressure. Circulation 2011;124:772-8.

[24] Santangeli P, Hamilton-Craig C, Dello Russo A, Pieroni M, Casella M, Pelargonio G, et al. Imaging of scar in patients with ventricular arrhythmias of right ventricular origin: cardiac magnetic resonance versus electroanatomic mapping. J Cardiovasc Electrophysiol 2011;22:1359-66.

[25] Santangeli P, Zado ES, Supple GE, Haqqani HM, Garcia FC, Tschabrunn $\mathrm{CM}$, et al. Long-term outcome with catheter ablation of ventricular tachycardia in patients with arrhythmogenic right ventricular cardiomyopathy. Circ Arrhythm Electrophysiol 2015;8:1413-21.

[26] Ahmed J, Smith WM, Ruygrok PN, Occleshaw C. Right ventricular morphology on catheter angiography: variations and its implications for the diagnosis of arrhythmogenic right ventricular cardiomyopathy. Heart Lung Circ 2012;21:700-5. http://dx.doi.org/10.1016/j. hlc.2012.06.002. 